\title{
Drift compression of an intense neutralized ion beam
}

P. K. Roy, S. S. Yu, E. Henestroza, A. Anders, F. M. Bieniosek, J. Coleman,

S. Eylon, W. G. Greenway, M. Leitner, B. G. Logan and W. L. Waldron

Lawrence Berkeley National Laboratory, I Cyclatron Road, Berkeley, CA-94720, U.S.A

D. R- Welch and C. Thoma

ATK Mission Research, Albrquerque, New Mexico 87110-3946, U.S.A

A. B. Sefkow, E. Gilson, P. G. Efthimion and R. O. Davidson

Princaton Plasma Physics Laboratory, New Jersey 08549-0451, U.S.A.

(Dated: September 1, 2005)

\begin{abstract}
Longitudinal compresgion of a teilored-velocity, intense neutralized ion beam hng been demonstrated. The compression tnlkes place in a 1-2 m drift section filled with plasma to provide spacecharge geutralization. An induction cell produces a hend-to-tail velocity ramp that longitudinally compresges the neutralized beam, enhancing the bean peak current by a factor of 50 and producing a pulse duration of about 3 ns. This measurament has been confirmed independently with two different dingnostic zystems.
\end{abstract}

PAOS numbers: 28.52, 28.17, 29.20; 28.27, 28.52, 41, 52, 94.20, 84.30

The simultaneous iransverse and longitndinal compression of an ion beam is required to achieve the high intensities necrsbary to crente high energy density matter and fusion conditions. A recent driver study for inertial fusion, for example, requires that $120 \mathrm{Bi}^{\circ} \div$ beams, each of $\sim 50 \mathrm{~kJ}$, be focused onto the terget with small focal spot sizes of radius $<2 \mathrm{~mm}$, and pulse lengths of $\sim 10 \mathrm{~ns}[1,2]$. Simulatione showed that these amall spot sizes could be achieved with plasma neutralization $[3,4]$. A scaled experiment, the Neutralized Transport Experiment (NTX) [5-8], subsequently demonstrated that an un-neutralized beam of geveral centimeter radius can be.compressed transversely to $\sim 1 \mathrm{~mm}$ radius when charge neutralization by backgromond plasma electrons is provided, in quantitative agreement with the simulation $[9,10]$.

Longitudinal compression of space-charge-dominated beams has been studied extensively in theory and simula. tions [11-16]. The compression is initiated by imposing a linear head-to-tail velocity tilt to a drifting beam. Longitudinal space-charge forces limit the bearn compression ratio, the ratio of the initial to final current, to about ten in most applications. An experiment with five fold compression bas been reported [17].

Recent theoretical models and simulations predict that much higher compression ratios $(\leq 100)$ could be achieved if beam compression takes place in a neutralized drift region $[18,19]$. To experimentally study the effects of plasma zeutralization on beam compression, the Neutralized Drift Compression Experiment (NDCX) was constructed at the Lawrence Berkeley National Laboratory. Figure 1 shows a schemntic of the NDCX benm line. The NDCX experiment uses the same front end as the earlier NTX expcriment. It consists of a $300 \mathrm{keV}, 25$-milliamp $\mathrm{K}^{+}$beam from an alumino silicate source powered by a Marx generator. Four pulsed quadrupoles magnets used in NTX to control the beam envelope (beam radius and convergence angle) are retained for the present exper-

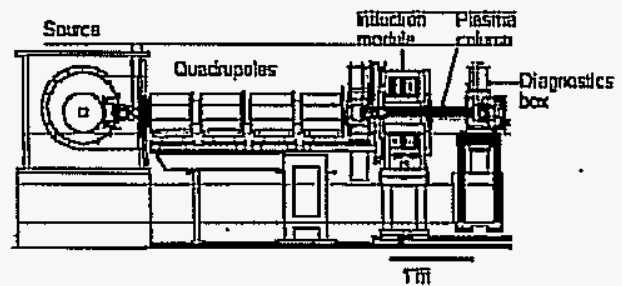

IIG. 1: Schemntic of the NDOX experimental setup.

iments on NDCX. To provide the head-to-tail velocity tilt, an induction module with variable voltage waveform is placed immedintely downstream of the last quadrupole magnet. This is followed by a neutralized drift section which consists of a one -meter-long plasma column produced by an $\mathrm{Al}$ cathodic arc [20]. A diagnostic box is located at the downstream end of the planma column.

The beam produced from the source has a $6 \mu \mathrm{s}$ lat-top. The induction tilt voltage 'carves' out a 300 ns segment of the flat-top which compresses longitudinally as it drifts through the plasma column. The final compressed beam is mensured in the downstream diagaostic bor.

The induction cell consists of 14 independently-driven magnetic cores in a pressurized gas $\left(\mathrm{SF}_{B}\right)$ region that is separated from the vacuum by a conventional high voltage insulator. The waveforms applied to the 14 cores inductively add at the acceleration gap. Each core is driven by a thyratron-5witched modulator. Because the modulator for each core can be designed to produce different waveforms and can be triggered independently, a variety of waveforms can ba produced at the acceleration gap using the 14 discrete building blocks.

The plasme column is formed by two pulsed aluminum cathodic are sources located at the downstream ond. Each source is equipped with a $45^{0}$ open-architecture 


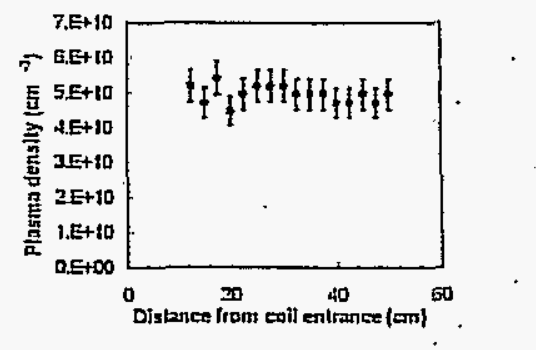

FIG. 2: Axial aluminum plasme density in the plasma column mensured with a movable probe.

macroparticle filter providing a flow of fully ionized aluminum plasma [21]. The two plnsma flows rvere pointed at an angle of $45^{\circ}$ towards the solenoidal column ( $\mathrm{HKG}, 7.6 \mathrm{~cm}$ diameter, and $1 \mathrm{~m}$ long). A significant fraction ( $>10 \%$ ) of the plasma enters the solenoid, and drifts practically unattenuated through the entire column (the rest of the aluminum plasma condenses at the wiall and thereby removed from the system). Figure 2 shows the plasma density measured with a biased probe within the plasma column. In most of the operating regimes, the plusma density is at least a factor of 10 higher than the bearn density. At the upstream end of the column, we have introduced a 'plasma stopper' consisting of two opposing dipoles of $\sim 1 \mathrm{kG}$ each, which inhibit the motion of plnsma upstream into the induction gap and quadrupole focusing sections. A second plasma column consisting of a meter-long ferro-electric plasma source that does not require solenoidal confinement has been constructed and is undergoing experimental tests.

A phototube diagnostic [22] is uged to measure beam pulse compression with and without neutralization by backgraund plasma. The optical system is based on a Hamamatsu phototube with fast (sub-ns) response which is coupled to a 500-MHz oscilloscope. The benm pulse is measured by using the phototube to collect the optical photon flux from an aluminum oxide scintillator placed in the path of the beam. The time response of the scintillntor is fust enough to make megsurements on a nanosecond time scale. Small amounts of stray light emitted by the plosma over long periods of time ( $100 \mathrm{~s}$ of $\mu \mathrm{s}$ ) can drain the bias charge in the phototube's internal power supply, and thus spoil the gain of the phototube during the beam pulse. This background plasma light is blocked from entering the phototube by an electro-optic gated shutter (Displaytech) that opens just before the bearn pulse arrives at the scintillator. The scintillator itself is not sensitive to low-energy plasma electrons. As a result, we have been able to obtain beam pulse compression data with minimal interference from the neutralizing plasma.

A second diagnostic, a Furaday cup, is used for measurements of the ciurent. The Faraday cup is specially designed [23] to function in a plasma environment and to hnve good time resolution. It consists of bole plates with hole gizes comparable to the Debye Iength, in order

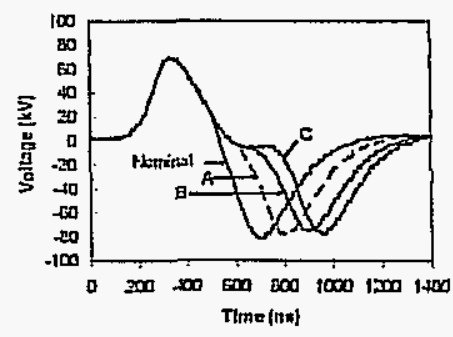

FIG. 3: Induction module valtage wavelorms produced by varying the tirning of the modulatorg.
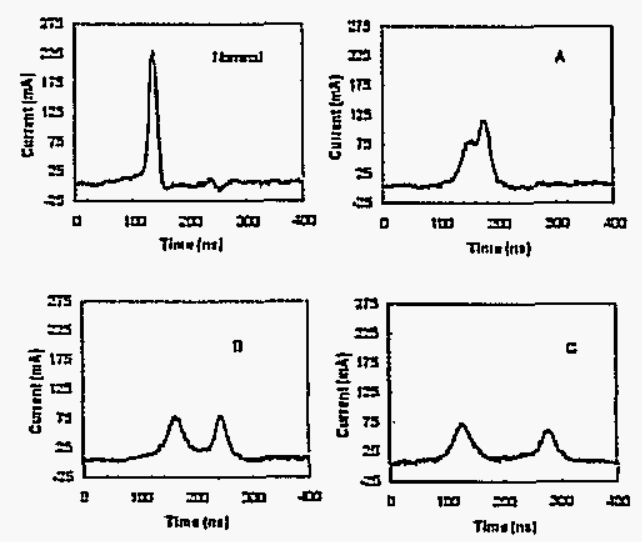

FIG. 4: Neutralized drift-compreased beam current with the voltage vaveforms in Fig.3.

to prevent plasma from entering into the cup. The cup geometry and external circuitry are optimized to assure fast time response ( $\sim \mathrm{Bnz})$.

When the tilt voltage waveform is turned on, beam bunching is observed in the downetream diagnostic box. The degree of bunching, as well as the pulse ehape, shown in Fig. 4, is clearly correlated with the voltage waveform, shown in Fig. 3. Theory specifies the ideal voltage waveform required to produce an exactly linear (versus z) velocity ramp $[18,19]$. The tilt core waveform was optimized to obtain a rather close approximation to the ideal wavelorm as shown in Fig. 5 . For a given voltage waveform, the position of maximal compression is changed as the beam energy is varied. A scan in beam energy demonstrates this behavior and is shown in Fig. 6.

Thus the maximum compresiion is observed by fine tuning the bean energy to match the voltage weveform and pracisely position the longitudinal focal point at the dingnostic location. This is shown in Fig. 7. The 50 fold compression ratio, [see Fig. 7(b)], is obtained by taling the ratio of the signal with tilt voltage on (with compression) to the signal with tilt voltage off (without compression) [see Fig. 7(a)]. A similar result is mensured with the Fararday cup as shown in Fig. 8. The LSP 


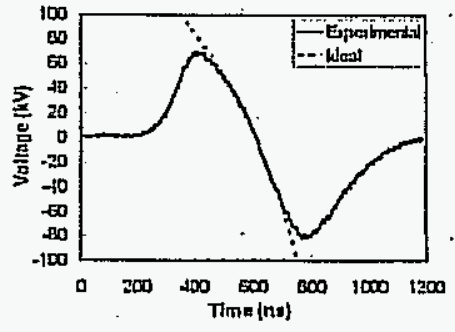

FIG. 5: Experimentally optimized and jueal induction madule voltage waveforms.

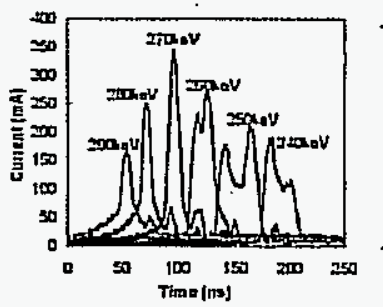

FIG. 6: Compressed beam current pulsos using a nominal tilt care voltage waveform as the beam energy is varied.

simulation under these experimental conditions is shown in Fig. 9.

The strong effects of neutralization are evident by comparing the compression ratio with the plasma turned on and off. Figure 10 shows that the pealk current is sigrificantly reduced when the plasma is turned oIf. LSP simulations under similar conditions show qualitatively similar results.

Optical imaging has also been deployed to measure the transverse beam size as a function of time. We are able
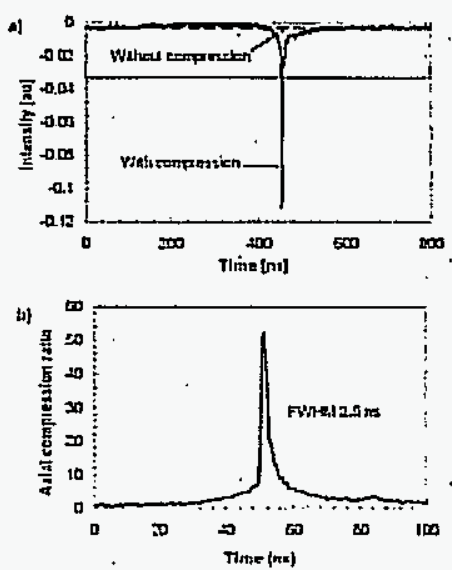

FIG. 7: (a) Measurements of benm signal using the phototube diegnostic for neutralized non-compressed, and neutralized compressed beams, and (b) compression ratio obtained from the mensurements,

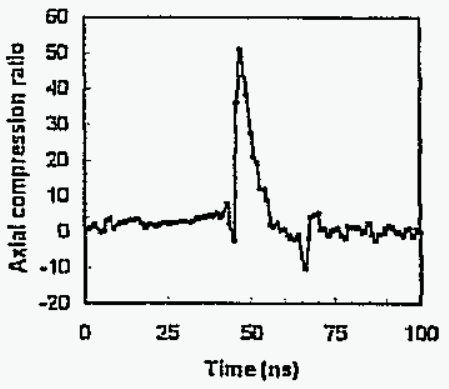

FIG. 8: Neutralized drifl compression mensurement using the Faraday cup.

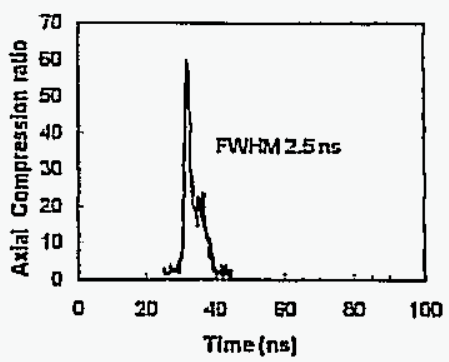

FIG. 9: LSP simulntion of neutralized drift compression of a $25 \mathrm{~mA} \mathrm{KC}^{+}$beam using an ideal velocity tilt core waveform.

to measure the images with a 1 ns time resolution. It is interesting to observe that the transverse spot size is larger at the point of maximal compression, as shown in the Figs. 11(a) and 11(b). This feature is due to timedependent defocusing effects occurring at the induction gap. The theory and simulations of this effect will be reported elsewhere.

Theory predicts that the nature of benm compression is strongly dependent on the drift length [18). As the length is increased, the compression is more sensitive to the degree of neutralization. It is also more sensitive to the intrinsic longitudinal temperature of the ion beam.

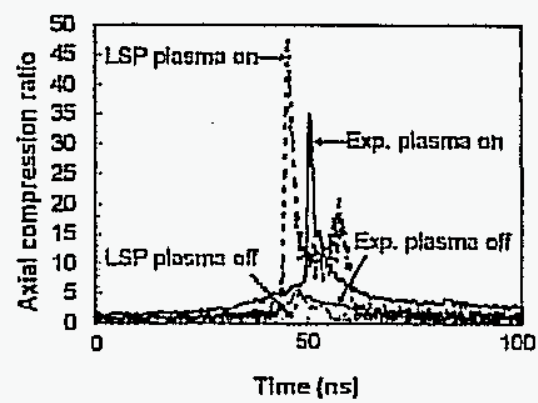

FIG. 10: Experimental data and LSP simulation of bent compression with neutralization (plasma source on) and without neutralization (plasma source off). 

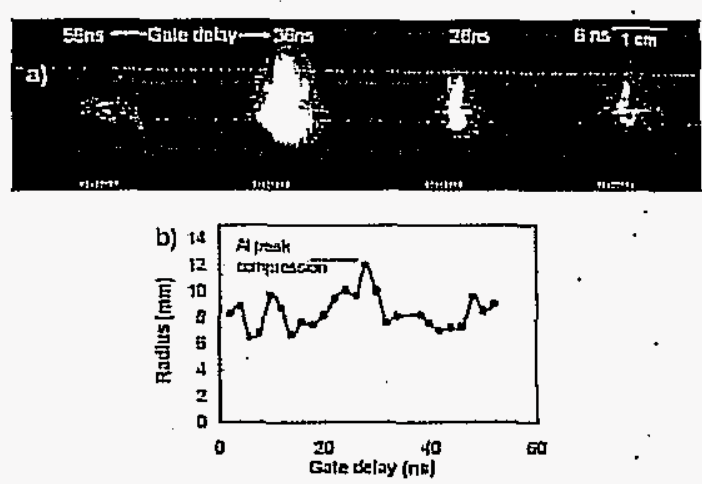

FIG. 11: Transverse images of neutralized comipressed benm: (a) optical profile, and (b) beam radius. Note that the beam radius has increased at the point of maximum compression.

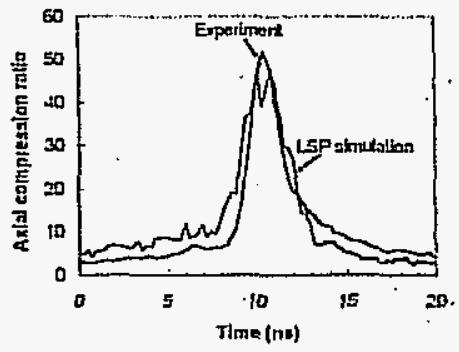

FIG. 12: Comparison of beam compression between experiment and LSP simulation for the 2-m long plnsme column.

Finally, if there arc any instabilities, e.g. two-strenm, they may become evident with longer drift lengths. Although theory predicts two-stream effects to be benign, an experimental confirmation would be desirable.

For the above reasons, we have performed additional experiments with the drift length with plasma extended to two meters. We were able to recover the 50-lold compression in the 2-m experiment as shown in Fig. 12. The corresponding LSP simulation is also shown. If the compressed pulse length $t_{p}$ were dominated by the longiturdinal beam temperature $T_{1}, t_{p}$ would be approximately given by

$$
t_{p}=\frac{L}{v_{l}^{2}} \sqrt{\frac{2 k T_{l}}{M}}
$$

which yield roughly 3 as for $T_{l}<1 \mathrm{eV}$, where $v_{l}, L_{2}$ $M$ and $k$ are the mean longitudinal beam velocity, drift length, mass of ion and Boltzmann constant, respectively.

On the basis of this two-meter experiment we conclude that: 1) the degree of charge neutralization is sufficient to achieve $\mathbf{5 0}$ fold loggitudinal compression while avoiding space-charge blaw-up of the beam for the experimental configuration investigated; 2) the intrinsic longitudinal temperature is $<1 \mathrm{eV}$; and 3 ) no collective instabilities have been observed.

\section{A.cknowledgments}

This Research was oupported by the U.S. Department of Energy under Contract No. DE-AC02-05CH11231 with the Lawrence Berkeley National Laboratory, and Contract No. DE-AC02-76CH03073 with Princeton PJagma Physics Laboratory. We thank Dr. A. Friedman, Dr. J. Barnard, Dr. C. Celata, Dr. I. Kaganovich, Dr. E. Lee, Dr. P. Seidl and Dr. W. M. Sharp for useful discussions and comments. Thanles also to Mr. D. Baen and Mr. D. L. Vanecek for useful technical assistance.
[I] S. S. Yu et al,,Nucl. Ingtrum. Moth. Phy. Res. A 544, 284 (2005).

[2] W. M. Sharp et al., Fusion Sci. Technol. 43, 393 (2003).

[3] D. R. Welch et al., Nucl. Ingtrum. Meth. Phys. Res. A 464,134 (2001).

[4] W. M. Sharp et al., Nucl. Fution 44, 221 (2004):

[i] S. S. Yu et al., in Proc. of the 2003 Particle Accelerrtor Gonf., edited by J.Chew, (IEEE, 2003), p: 98.

[6] E. Henestrazn, et al., Fhys. Rev. ST Accel. Beams 7, 083501 (2004).

[7] P. K. Roy et al., Pliys. of Plosmas 11, 2890 (2004).

[B] B. G. Iogan et al., Nucl. Fusion 45, 131 (2005).

[9] C. Thomn et al., Phys. of Plasmas 12, 043102 (2005).

[10] P. K. Roy et al., Nucl. Ingtrum. Meth. Phy. Res. A 544, 225 (2005).

[11] D. D.-M. Ho et al., Particle Accelerators 35, 15 (1991).

[12] T. Fillouchi et al., Phys. of Plasmas 9, 3476 (2002).

[13] M. J. I. de Hoon et al., Phys. of Plasmag 10, 855 (2005).

[14] H. Qin et al., Phys. Rev. ST Accel. Beams $T, 10 d 201$
(2004).

[15] R. C. Duvidsan and H. Qin, Phys. Hev, ST Accel. Beams 8, 064201 (2005).

[16] W. M. Sharp et al., Nucl. Instrum. Meth. Phys. Res. A 544,398 (2005).

[17] W. M. Fnwley et al., Phys. Plasmas 4, 880 (1997).

[18] D. R. Welch et al., Nucl. Instrum. Meth. Phys. Res. A 544,236 (2005).

[19] G. Thoma et al., Proc. of the 2005 Particle Accelentor Conf., in press (IEEE, 2005).

[20] A. Anders and G. Y. Yushkov, J. Appl. Phye. 91, 4824 (2002).

[21] A. Anders and R. A. MacGill, Surf. Coat. Technol. 138$134,96(2000)$.

[22] F. M. Bieniosels et al., Nucl. Instrum. Meth. Phy. Res. A 544, 268 (2005).

[23] A. Selkow et al., Proc. of the 2005 Particle Accelerator Conf, in press (IEEE, 2005). 